\title{
Norois
}

Environnement, aménagement, société

$191 \mid 2004 / 2$

Les types de temps

\section{Brume sèche, brume de poussière, chasse-sable et tempête de sable}

Des types de temps spécifiques des régions sèches

\section{Zeineddine Nouaceur}

\section{(2) OpenEdition}

\section{Journals}

\section{Édition électronique}

URL : https://journals.openedition.org/norois/1188

DOI : $10.4000 /$ norois. 1188

ISBN : 978-2-7535-1539-0

ISSN : 1760-8546

\section{Éditeur}

Presses universitaires de Rennes

\section{Édition imprimée}

Date de publication : 1 mars 2004

Pagination : 121-128

ISBN : $978-2-86847-977-8$

ISSN : 0029-182X

\section{Référence électronique}

Zeineddine Nouaceur, "Brume sèche, brume de poussière, chasse-sable et tempête de sable », Norois [En ligne], 191 | 2004/2, mis en ligne le 09 septembre 2008, consulté le 13 janvier 2022. URL : http:// journals.openedition.org/norois/1188; DOI : https://doi.org/10.4000/norois.1188

Ce document a été généré automatiquement le 13 janvier 2022.

() Tous droits réservés 


\title{
Brume sèche, brume de poussière, chasse-sable et tempête de sable
}

Des types de temps spécifiques des régions sèches

\author{
Zeineddine Nouaceur
}

\section{La typologie des types de temps lithométéoriques}

1 Le lithométéore est défini par l'atlas international des nuages (OMM, 1956) comme un météore consistant en un ensemble de particules dont la plupart sont solides et non aqueuses et qui se trouvent plus ou moins en suspension dans l'atmosphère ou soulevées par le vent. Souvent assimilés à un vent de sable, les lithométéores représentent cependant une série de types de temps bien différenciés :

- Les brumes sèches et les brumes de poussière correspondent à une situation postérieure à un soulèvement de particules fines.

- Les chasses-sable et les tempêtes de sable caractérisent un phénomène qui associe pleinement la dynamique éolienne et le transport des particules.

2 Les définitions de ces phénomènes régionaux sont relatées par le recueil des codes météorologiques (OMM, 1973) et se présentent ainsi :

- Brume de poussière : (photo 1) (S), « poussières en suspension dans l'air d'une manière généralisée, non soulevées par le vent au point d'observation ou à ses alentours au moment de l'observation ». La brume de poussière est mentionnée par l'observateur, lorsqu'un dépôt de poussière éolienne est visible au sol.

- Brume sèche : $(\infty)$ : il n'existe pas de définition précise pour ce type de lithométéore. La brume sèche possède cependant les mêmes caractéristiques que la brume de poussière, mais en est différente par l'absence de dépôt de poussière au sol.

- Chasse-sable : (\$) « poussière ou sable soulevés par le vent au point d'observation ou à ses alentours au moment de l'observation, mais absence de tourbillon et pas de tempête de poussière ou de sable observée ".

- Tempête de sable (photo 2) : (-S $\rightarrow$ ) « tempête de poussière ou de sable observée au moment de l'observation en vue du point d'observation, ou à ce point pendant l'heure précédente ». 
Photo 1 : Brume de poussière à Nouakchott le 2 janvier 1994 (carrefour ASECNA à $9 \mathrm{~h}$ 45, cliché Z. N.)

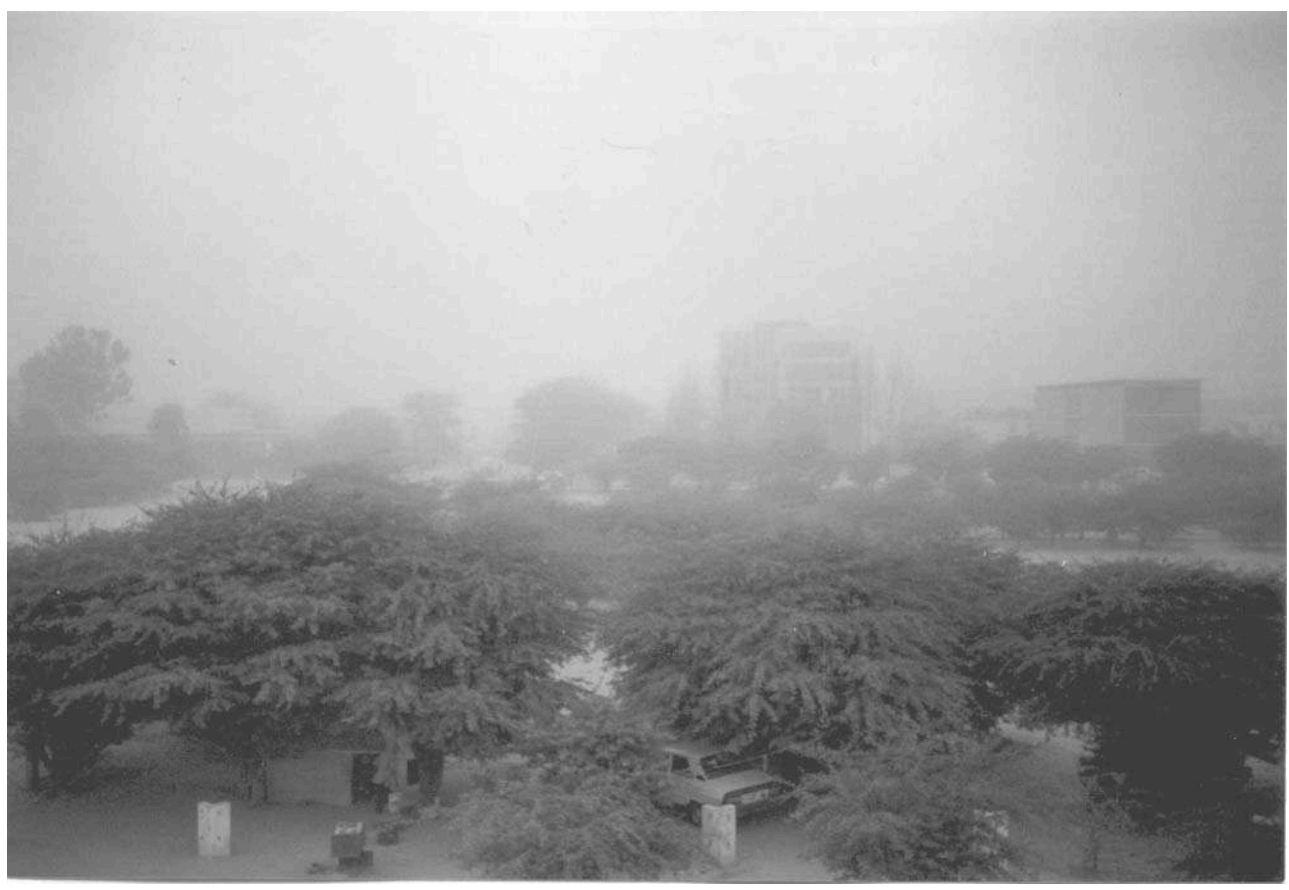

Photo 2 : Tempête de sable à Nouakchott le 27 janvier 1993 à 15 heures. La réduction de la visibilité est plus importante qu'en période de brume (cl. Z. N.)

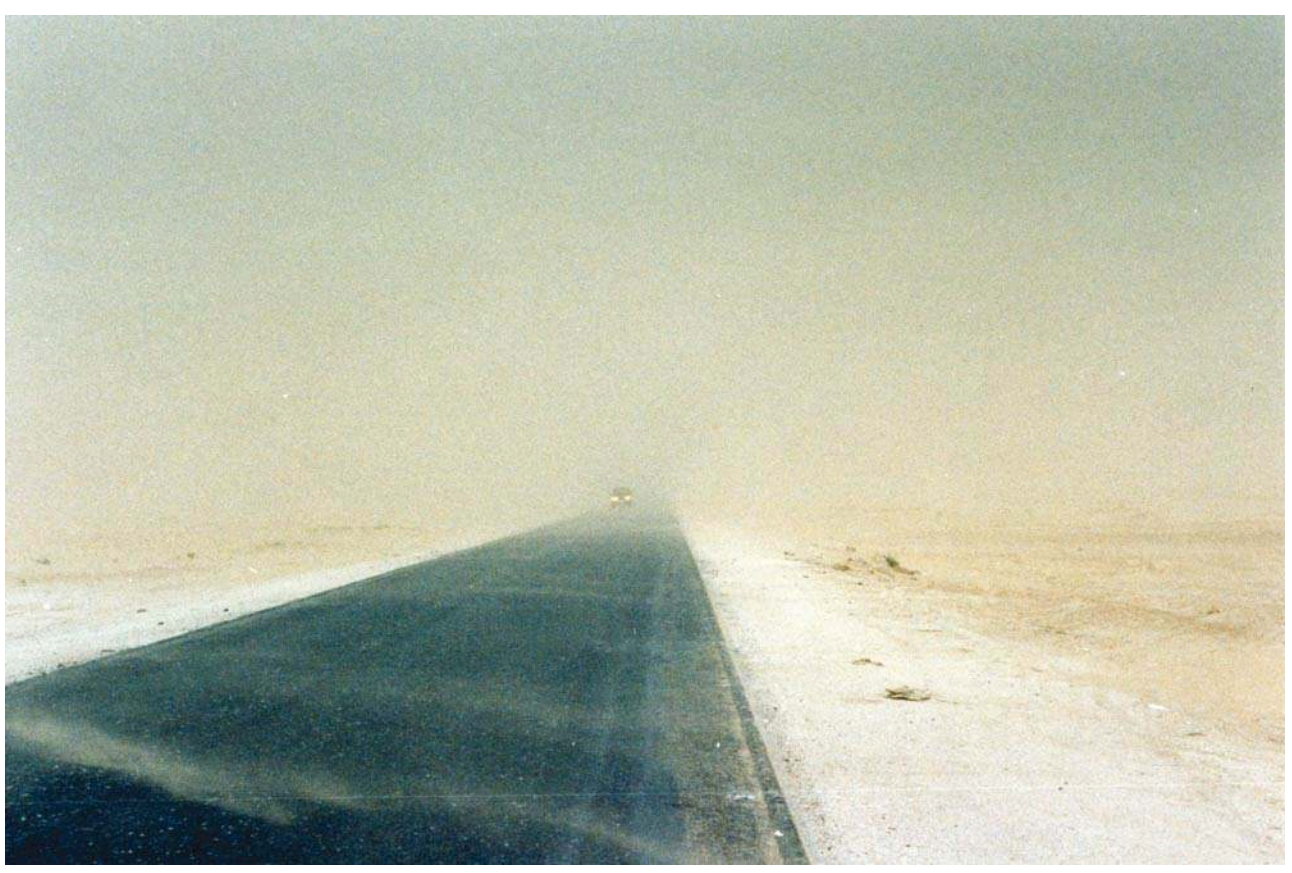

Les phénomènes locaux se caractérisent par une occurrence ponctuelle et se traduisent par des manifestations de tourbillons thermiques ou dynamiques, ou de murs de sable qui représentent les formes les plus simples des lithométéores mais qui restent assez spectaculaires. 
Les tourbillons de poussière ou de sable sont : « un ensemble de particules de poussière ou de sable, accompagné parfois de petits débris, soulevés du sol sous forme d'une colonne tourbillonnante et de hauteur variable, à axe sensiblement vertical et de faible diamètre ». Lorsque le tourbillon est thermique, il est induit par une instabilité due à un échauffement du sol. Quant il est dynamique, il est généré par l'existence de deux courants de vitesses ou de directions différentes.

Le mur de sable (photo 3) n'est pas défini, seule sa formation est évoquée. Il constitue le bord antérieur d'une tempête de sable ou d'une invasion d'air froid.

6 L'observation de ces deux phénomènes reste assez rare aux abords immédiats des stations météorologiques.

Photo 3 : Mur de sable (haboob) aux environs de Kiffa en mai 1994 (cl. Z. N.)

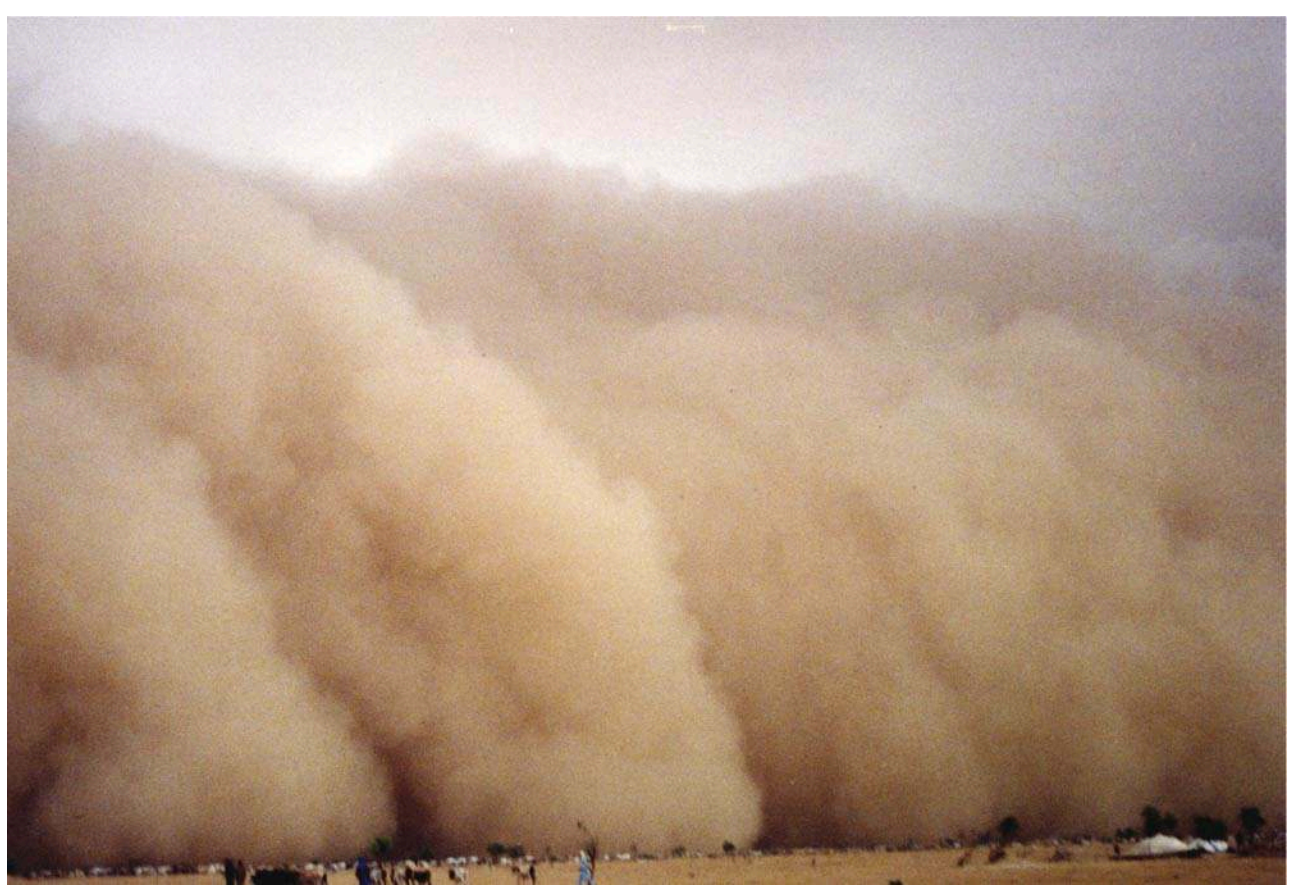

\section{La genèse des lithométéores}

7 Si la typologie du phénomène lithométéorique est complexe, la genèse de ces types de temps est tout aussi difficile à maîtriser. Deux facteurs majeurs conditionnent cependant l'apparition des lithométéores.

\section{Un facteur climatique}

Le premier maillon de la chaîne dans la formation des lithométéores est sans conteste le vent. Agent redoutable d'érosion, il agit selon un cadre multidirectionnel et intervient ainsi dans l'arrachage des sédiments depuis leur structure de base constituée par le sol. Il véhicule ensuite ces particules et les transporte sur plusieurs centaines et parfois même sur des milliers de kilomètres. Dans ce contexte, la dynamique éolienne revêt une part primordiale et son action est différentielle sur le substrat lithologique et pédologique. 
9 La turbulence de l'atmosphère et les systèmes perturbés représentent l'autre élément climatique qui intervient dans l'élaboration des lithométéores. Ils favorisent le soulèvement des particules à différentes échelles spatiales. Cette différence nous permet de distinguer les phénomènes locaux, tourbillons, mur de sable et chasse-sable, des phénomènes régionaux qui s'expriment en nuages de sable et de poussière. Cette différence d'échelle est une autre problématique de la genèse des lithométéores.

\section{Un facteur lithologique et pédologique}

10 L'action éolienne est différentielle sur le substrat pédologique. Elle est fonction de la taille de la particule libre, de la structure et de la stabilité d'un sol. Elle est aussi liée à la force $d u$ vent nécessaire pour prendre en charge les éléments fins. Le spectre granulométrique éolien est ainsi varié, il oscille entre les argiles, les limons et les sables, auxquels s'associent des éléments de nature organique (débris végétaux, pollens, micro-organismes divers).

Une fois le substrat fragilisé ou débité en fines particules, la prise en charge du matériel fin est fonction de la force du vent.

- Une première intervention de l'agent éolien concerne la déflation directe des substrats très aptes à l'érodibilité.

- Le prélèvement par abrasion sur les roches cohérentes fragilisées (corrasion) constitue un deuxième mode d'extraction des éléments fins. La corrasion du quartz qui s'exerce sur les substrats est qualifiée d'un effet de « râpe » (Coudé-Gaussen, 1991, 1994). C'est une action particulièrement érosive durant les chasses-sable et lors des tempêtes. Elle permet en outre de dégager des éclats ou des fragments de roches de petite taille, qui alimentent les poussières et qui sont reconnaissables à leur forme anguleuse. Obéissant au mouvement éolien, le quartz prélève des substrats les particules argileuses et les rend sensibles à la déflation. Ce matériel se trouve ainsi intégré à la poussière, il y subit le processus d'amenuisement déjà énoncé et évolue en une fine fraction argileuse.

- Enfin, le vannage de la fraction fine des sables mobiles et des sols est l'ultime action éolienne efficace.

L'intensité des processus initiaux élucide la production de la fraction fine mobilisable par le vent. Le vannage ininterrompu de tous les substrats qui sont exposés à l'action éolienne pose le problème du renouvellement incessant de la poussière fine qui alimente les lithométéores. Ainsi, la pellicule rousse qui se dépose par temps lithométéorique participe par sa mobilisation et sa nature à la production de poussières. L'expérimentation en soufflerie menée par Coudé Gaussen (1991) sur les sables désertiques a permis d'apporter des éléments de réponse, sur l'efficacité des grains de sable mobilisés au sol (processus de la comminution). La pellicule rousse doit sa couleur à de minuscules dépôts d'argiles colorés par le fer et piégés dans les creux et les caries qui alimentent leur surface. Ce matériel quartzeux et limono-argileux représente un résidu sableux déposé à l'issue des tempêtes de sable et lors de l'occurrence des brumes de poussière. Avec une médiane comprise entre 35 et $105 \mu \mathrm{m}$, les grains de sable et de poussière présentent des caractères micromorphologiques issus de leur remaniement incessant (Nouaceur, 1999). Ce mouvement permanent à la surface des sols est à la base d'une action abrasive continue. 


\section{L'observation et l'identification des différents types de lithométéores}

\section{L'observation au sol}

13 L'appréciation des lithométéores se fait à partir d'observations au sol, ce procédé est donc étroitement lié à la compétence et à l'évaluation des agents.

Dubief, en 1952, insistait déjà sur les problèmes de définitions des vents de sable et des vents de poussière. Il note à cet égard le rôle de l'observation, il précise: «Les observateurs ne possédant pas de critères précis pour savoir quand il est justifié de noter 'vent de sable' en sont réduits à leurs impressions personnelles. Celles-ci seront nécessairement différentes d'un individu à l'autre, variables pour l'un d'entre eux au cours de son séjour. Un nouveau venu aura tendance à noter trop de vents de sable ; un ancien à ne signaler que les tempêtes. À noter aussi que, dans les stations où les vents de sable sont des phénomènes courants, on sera tenté de négliger ceux de faible importance. En bref, ces observations sont entachées d'un coefficient personnel important. »

Cinquante années plus tard, l'évolution scientifique et l'avancée technologique n'ont guère amélioré la prise en compte du phénomène. Si d'autres critères permettent de renforcer l'exactitude des informations relatives aux lithométéores, recueillies dans les stations météorologiques, elles n'en sont pas moins critiquables (cas de la réduction de la visibilité).

\section{Lithométéore et réduction de la visibilité}

16 L'une des conséquences les plus immédiates de l'occurrence des lithométéores est sans nul doute la réduction de la visibilité qui accompagne ces types de temps, et qui est conséquente au soulèvement et à la suspension des sables et des poussières dans l'atmosphère. La réduction de la visibilité est proportionnelle à la concentration d'éléments fins dans l'atmosphère, qui varie fortement suivant le type et l'intensité du phénomène. En présence des types de temps de chasse-sable, la visibilité est peu altérée; elle est plus réduite en présence des tempêtes de sable (photo 2) et lors des brumes sèches et des brumes de poussière. Les brumes ne sont d'ailleurs mentionnées que si la visibilité est inférieure à $5 \mathrm{~km}$.

17 La densité de matériel pris en charge par les vents est variable non seulement d'un phénomène à l'autre, mais aussi suivant son intensité. Dans ces conditions, il est très difficile d'identifier les types de lithométéores à travers la masse de poussière et de sable mobilisée, comme il est presque impossible de dégager une granulométrie typique de chaque phénomène. Il est aussi très difficile et très délicat d'établir un seuil moyen de réduction de la visibilité lié à chaque phénomène. La variabilité spatio-temporelle des paramètres qui conditionnent l'apparition des lithométéores explique cette difficulté.

18 Dans leur ensemble, ces phénomènes limitent la visibilité suivant des seuils horaires et journaliers compris entre $100 \mathrm{~m}$ et $5 \mathrm{~km}$. La figure 1 illustre cette particularité : on remarque qu'à l'échelle des différentes stations, mais aussi des mêmes phénomènes lithométéoriques, le seuil de réduction de la visibilité est considérablement différent d'une journée à l'autre. 
19 Figure 1: La réduction de la visibilité en fonction des différents types de lithométéores (situation au mois de janvier 1993 à 12 heures, dans les stations mauritaniennes de Nouakchott et Néma). Bs, brume sèche. Bp, brume de poussière, Chs, Chasse-sable, Ts, Tempête de sable

Figure 1 : La réduction de la visibilité en fonction des différents types de lithométéores (situation au mois de janvier 1993 à 12 heures, dans les stations mauritaniennes de Nouakchott et Néma).
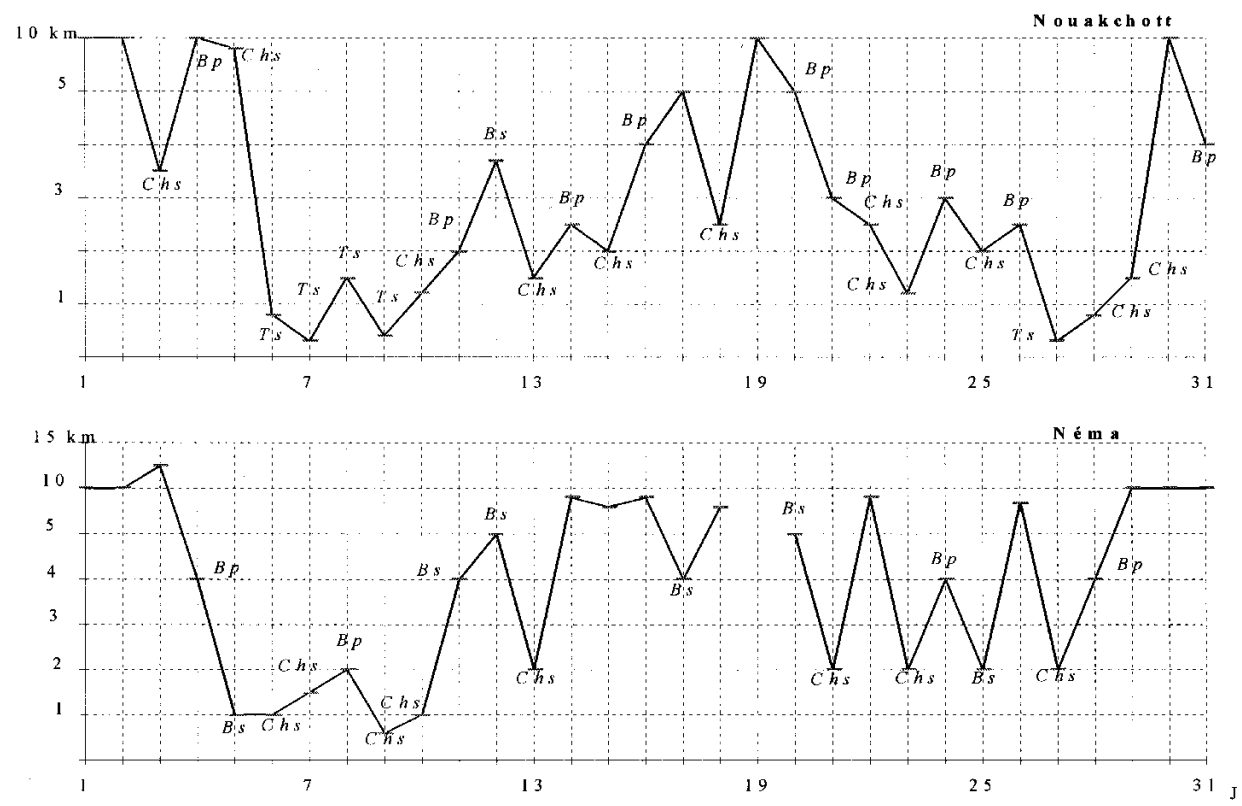

Bs, brume sèche. Bp, brume de poussière, Chs, Chasse-sable, Ts, Tempête de sable

Les images satellites prises lors de grands phénomènes régionaux plus connus sous le nom de nuages de poussière (photo 4) laissent mieux apprécier l'importance de la concentration dans l'atmosphère des particules pour la plupart terrigènes. Cette masse de poussière et de sable est cependant évaluée d'une manière très dissemblable d'une station à l'autre à travers la réduction de la visibilité qu'elle occasionne au sol. 
Photo 4 : Expulsion de poussières vers l'Atlantique, conséquence de l'occurrence de types de temps lithométéoriques sur l'Afrique de l'Ouest. Image satellite (visible), « Météosat » du 8 mars 1998

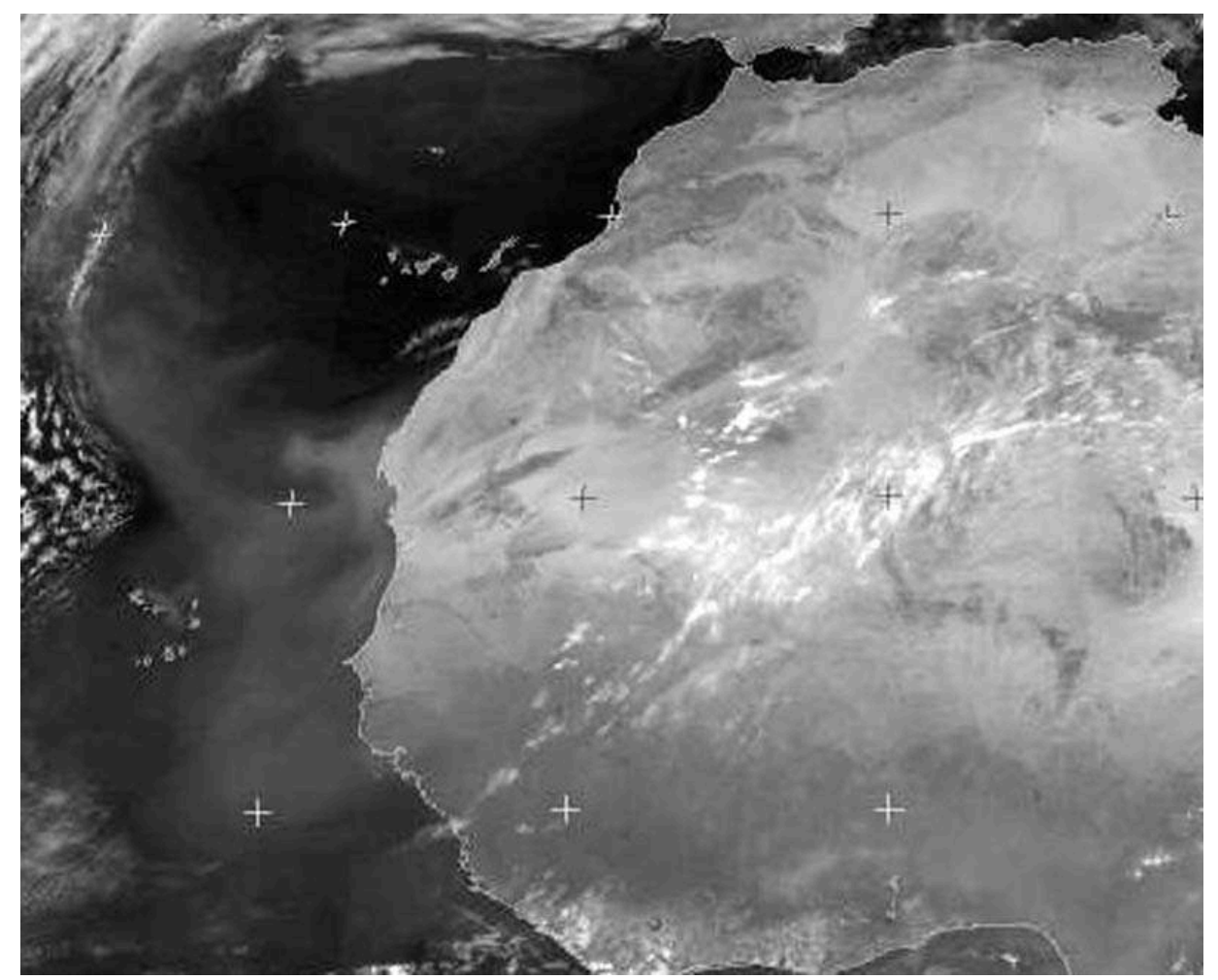

\section{Des méthodes d'observations subjectives et des définitions incomplètes}

21 L'appréciation du phénomène lithométéorique est assez subjective. Les définitions du manuel des codes météorologiques sont incomplètes, dans la mesure où la description du type de temps reste insuffisante puisqu'elle ne fait pas état du phénomène déclencheur: la force du vent et la turbulence dynamique ou thermique. La force du vent pourrait constituer un point essentiel de distinction. Ainsi, les tempêtes de sable ne se déclenchent que lorsque les vents atteignent des vitesses généralement supérieures à $6 \mathrm{~m} / \mathrm{s}$, tandis que l'observation des brumes sèches et de poussière ne se fait que par temps relativement calme pour permettre la suspension durable des particules fines. Cette problématique est justifiée par la diversité des lithométéores et des phénomènes déclencheurs. Cependant, la vitesse du vent est un point crucial considéré comme précurseur au déclenchement du phénomène et notamment lors des chasses-sable et des tempêtes de sable.

L'appréciation de la visibilité au sol est évaluée dans les stations météorologiques aux heures synoptiques d'observation. Elle correspond à la distance maximale à laquelle un observateur peut identifier un repère, dans la direction où l'observation est la moins bonne. Elle ne permet pas non plus de spécifier avec exactitude le type de lithométéore. Un lien paraît cependant bien évident entre l'occurrence des lithométéores, la réduction de la visibilité et la concentration en particules de l'atmosphère. Ce qui est moins évident par contre, c'est de pouvoir identifier le phénomène en imposant des seuils de réduction de visibilité qui attestent de taux précis de concentration de particules dans l'atmosphère. Dans ce domaine, la variabilité spatio-temporelle des 
lithométéores représente une entrave certaine et même l'utilisation d'appareils de mesure très sophistiqués ne pourra donner qu'une réponse très ponctuelle dans le temps et dans l'espace.

Ces quelques remarques évoquent la complexité d'un phénomène pluridimensionnel mis en évidence ces dernières années par l'imagerie satellitale (photo 4), ce qui permet de traduire sa réelle dimension et de suivre son évolution et sa progression d'une région à l'autre en Afrique de l'Ouest.

\section{Lithométéore et sécheresse climatique sahélienne}

Le milieu aride et semi-aride est réputé pour l'intensité de la morphogenèse mettant en jeu les actions mécaniques d'érosion. Ces actions contribuent à une intense désagrégation granulaire et à une fragmentation superficielle de la roche. L'appauvrissement de la flore provoqué par les sécheresses prolongées qui ont sévi au Sahel a accentué l'impact des actions mécaniques d'érosion contribuant ainsi à altérer la cohérence des sols. Ces dispositions se répercutent sur l'activité biologique qui devient plus réduite, ce qui minimise la fraction organique présente dans les sols et augmente ainsi leur érodibilité. L'érosion éolienne augmente exponentiellement lorsque le couvert végétal diminue (Valentin, 1994) et les pertes en terre dues au vent sont multipliées par quatre, lorsque ce couvert passe de 40 à $25 \%$ (Fryear, 1985). La conséquence visible d'un tel bouleversement est sans nul doute l'augmentation sensible de la disponibilité du matériel fin qui forme les lithométéores.

Tous les observateurs scientifiques s'accordent pour établir un lien étroit entre la baisse de la pluviométrie au Sahel et l'augmentation significative des lithométéores. L'évolution interannuelle établie pour les précipitations et les brumes de poussière enregistrées dans les stations mauritaniennes dévoile d'une manière éloquente cette liaison inverse. Les indices normalisés calculés à l'échelle nationale laissent apparaître une tendance très nette à la hausse des brumes dès l'année 1970 (fig. 2). 
Figure 2 : Indices normalisés des précipitations annuelles (histogramme) et des observations des brumes de poussière (courbe) pour la totalité des stations mauritaniennes (moyennes lissées sur cinq ans pour la période 1951-1990)

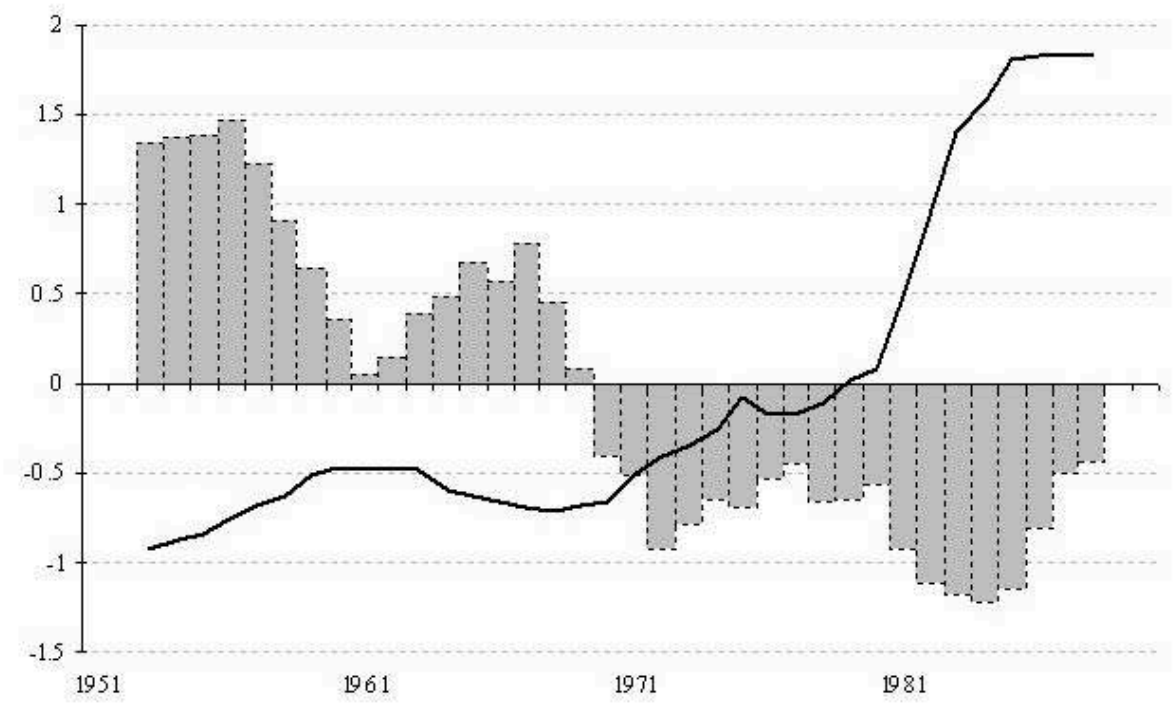

\section{Lithométéore et évolution du climat}

La tendance générale à l'augmentation de la fréquence des lithométéores observée dans tout le Sahel, depuis le début des années soixante-dix, a entraîné une réduction de la visibilité au sol. Cette réduction de la visibilité atteste à son tour d'une plus grande présence de particules diverses en suspension dans l'atmosphère.

Les conditions aérologiques stables (inversion des alizés, subsidence anticyclonique) et géographiques spécifiques (en fin de parcours des alizés continentaux, Irifi et Harmattan) de l'Afrique de l'Ouest, constituent des facteurs qui prédisposent cette région à une modification notable du bilan radiatif. De plus, les nuages de poussière qui arrivent dans cette région et ceux formés sur place sont contrariés dans leur progression vers l'ouest par les alizés maritimes (Nouaceur, 1994). Cette situation spécifique favorise la suspension durable des aérosols et des poussières (entre 6 et $7 \mathrm{~km}$ d'altitude, Legrand, 1990) et augmente ainsi leur influence sur le rayonnement solaire. Ainsi, une particule de $0,5 \mu \mathrm{m}$ possède une vitesse de chute de l'ordre de $1 \mathrm{~m}$ par jour, soulevée à des altitudes de 6 à $7 \mathrm{~km}$ son temps de résidence sera plus important. L'augmentation du nombre d'observations de brumes de poussière depuis la fin des années soixante-dix, plus particulièrement dans les régions du Sud-Ouest sahélien, confirme cette situation originale. Cette présence permanente d'une couche uniforme de poussière modifie le bilan radiatif et thermique au sol par le rôle d'écran qu'elle joue (absorption de rayonnement et rediffusion des flux). L'impact thermique au sol s'exprime par une variation positive du flux net de nuit (augmentation des températures minimales).

L'évolution des températures minimales (au Mali, en Mauritanie et au Sénégal) apporte une réponse logique a ces récents bouleversements climatiques (Nouaceur, Sagna, 
1996). Une hausse spectaculaire des valeurs est observée durant la saison de préhivernage, qui est la période habituelle de l'intensification de l'occurrence des lithométéores (tableau 1). On peut donc comparer ce phénomène, à un cas typique de pollution par des effluents terrigènes.

Tableau 1 : Tendance des températures minimales $\left({ }^{\circ} \mathrm{C}\right)$ pour la période $1970-1990$, par pays (dixsept stations)

\begin{tabular}{|c|c|c|c|}
\hline & Saison fraîche & Pré-hivernage & Année \\
\hline Mauritanie & $+1,97$ & $+2,46$ & $+1,84$ \\
Sénégal & $+1,58$ & $+2,34$ & $+1,42$ \\
Mali & $+0,12$ & $+0,47$ & $+0,03$ \\
Totalité des stations & $+1,42$ & $+1,96$ & $+1,29$ \\
\hline
\end{tabular}

\section{Conclusion}

Les lithométéores représentent des types de temps caractéristiques des régions arides et semi-arides. Malgré l'existence d'une typologie variée, l'identification de ces phénomènes reste toujours liée à l'appréciation de l'agent observateur. Durant les trois dernières décennies, les régions sahéliennes ont été le siège d'une recrudescence sans précédent de ces types de temps (Ozer, 2000, Nouaceur, 1999). Cette tendance climatique est conséquente d'un bouleversement écologique qui caractérise toute la région. L'une des conséquences d'une telle situation est la modification du bilan radiatif de la partie occidentale de l'Afrique de l'Ouest. L'augmentation de la concentration des poussières et des sables dans l'atmosphère liée à une plus grande recrudescence des phénomènes lithométéoriques est la cause la plus plausible d'une tendance à la hausse très significative des minima de température dans la région.

\section{BIBLIOGRAPHIE}

COUDÉ-GAUSSEN (G.), 1991. - Les poussières sahariennes, Paris, Montrouge, AUPELF/UREF, John Libbey Eurotext, $485 \mathrm{p}$.

-, 1994. - « Les régions sources de poussières au Sahara », Sécheresse, n² 2, p. 134-141.

Dubief (J.), 1951. - Les vents de sable au Sahara français, Actions éoliennes Phénomènes d'Evaporation et d'Hydrologie Superficielle dans les Régions Arides, Colloques Internationaux, Centre National de la Recherche Scientifique, Alger, CNRS, XXXV, p. 47-57.

-, 1952. - Le vent et le déplacement du sable au Sahara, Alger, Travaux de l'Institut de Recherches Sahariennes, vol. 8, p. 123-164.

FRYREAR (D. W.), 1985. - « Soil cover and wind erosion », Transactions of the AmericanSociety of Agricultural Engineers, $n^{\circ}$ 28, p. 781-784. 
LEGRAND (M.), 1990. - Étude des aérosols sahariens au-dessus de l'Afrique à l'aide du canal à 10 microns de Météosat : visualisation, interprétation et modélisation, thèse de Doctorat d'État en Sciences Physique, Université des Sciences et techniques de Lille Flandre-Artois.

NouAceur (Z.), SAGNA (P.), 1996. - « Le réchauffement du climat en Afrique de l'Ouest (Mauritanie, Sénégal, Mali) », Publications de l'Association Internationale de Climatologie,Thessaloniki, vol. 9, p. 463-470.

NOUACEUR (Z.), 1994. - « Les variations spatio-temporelles et les causes de mauvaise visibilité en Mauritanie », Publications de l'Association Internationale de Climatologie, Thessaloniki, Maheras, vol. 7, p. 477-484.

-, 1999. - Évolution récente des lithométéores en Mauritanie, thèse de Doctorat, Université JeanMoulin Lyon III, $503 \mathrm{p}$.

OMM, 1956. - Atlas international des nuages, vol. I.

OMM, 1973. - Guide des instruments et des observations météorologiques, $\mathrm{n}^{\circ} 8$.

OZER (P.), 2000. - Les lithométéores en région sahélienne : un indicateur climatique de la désertification, thèse de Doctorat en Sciences Géographiques, Université de Liège, 312 p.

VALENTin (C.), 1994. - « Sécheresse et érosion au Sahel », Sécheresse, n 5, p. 191-198.

\section{RÉSUMÉS}

Depuis les grandes sécheresses climatiques sahéliennes, la hausse des fréquences d'observation des types de temps associés au soulèvement et au transport des poussières et des sables, connus sous le nom de "lithométéores", a suscité un intérêt scientifique sans cesse croissant. Cette recrudescence, observée depuis 1973 dans tout le Sahel, ne devrait pas cependant nous faire oublier que les premières études qui mentionnent ces types de temps remontent aux années cinquante (Dubief, 1951) et que ces phénomènes représentent une composante du climat régional, que les récentes sécheresses ont exacerbé.

Since the great Sahelian droughts of the 70's, the increase in the occurrence of weather types associated with dust and sand (litho meteors) displacement aroused an increasing scientific interest. However, the first studies about these weather types have been published since the 50's (Dubief, 1951). The weather types with litho meteor displacement are a component of the regional climate, and their importance grew during the recent droughts.

\section{INDEX}

Keywords : drought, environmental degradation, lithometeors, climate change

Index géographique : Sahel

Mots-clés : lithométéore, sécheresse, changement climatique

\section{AUTEUR \\ ZEINEDDINE NOUACEUR}

Université de Rouen 